\title{
A Review of Imaging in Psychiatry
}

\author{
J. Lagopoulos ${ }^{*}, 1,2,3$, P. Das ${ }^{1,2,3}$, O. Saether ${ }^{4}$ and G.S. Malhi ${ }^{1,2,3}$ \\ ${ }^{I}$ Discipline of Psychological Medicine, Northern Clinical School, University of Sydney, Australia \\ ${ }^{2}$ CADE Clinic, Department of Psychiatry, Royal North Shore Hospital, St Leonards, Australia \\ ${ }^{3}$ Advanced Research and Clinical Highfield Imaging (ARCHI), University of Sydney, Australia \\ ${ }^{4}$ Department of Neuroscience, Norwegian University of Science and Technology (NTNU), Trondheim, Norway
}

\begin{abstract}
The past decade has seen significant advances in imaging techniques used to measure structure and function in the human brain and many of these have direct relevance to psychiatry. Psychiatric disorders clearly pertain to brain function, however the underlying biology of these putative dysfunctions remains obscure. From a research perspective integrated use of novel imaging techniques in conjunction with clinical assessments now affords an opportunity to advance our understanding of the pathophysiology of psychiatric conditions by allowing the in vivo investigation of abnormalities of brain function as they relate to changes in cognition, mood and behaviour. Whilst most of these imaging techniques are still very much entrenched in the research domain, it is important that clinicians understand the benefits of these new technologies while also appreciating their limitations. These imaging techniques are constantly evolving and hold significant potential to one day provide clinical insights that inform diagnosis and management.
\end{abstract}

Keywords: Neuroimaging, mood disorders, schizophrenia, PTSD, psychiatry, PET, fMRI, MRS.

\section{INTRODUCTION}

Technological advances in physics and computing along with a strong desire to understand the brain in terms of its structure and function have contributed to the development of sophisticated technology which permits an unprecedented ability to dissect the 'working brain' and better understand its neurobiology [1]. Progress in the field has been accelerated through iteration between an increasing knowledge base and methodological developments. The examination of diffuse processes such as metabolism and blood flow using Positron Emission Tomography (PET) has evolved to encompass receptor-imaging studies that map neuronal localisation within the brain. In the rapidly growing field of cognitive neuroscience, functional Magnetic Resonance Imaging (fMRI) is proving to be a useful tool that in combination with sophisticated signal processing and advanced statistical and visualisation equips investigators with the ability to probe the mind and secure findings to the anatomy of the brain. Similarly, Magnetic Resonance Spectroscopy (MRS) has opened a neurochemical portal that permits in vivo sampling of chemical activity. Collectively, in addition to better understanding the architecture of the brain and its changes through neurodevelopment and neurodegeneration, these technologies have revealed meaningful associations between brain biology and human behaviour, both in health and disease. Coupled with more recent developments, which permit the partitioning of grey and white matter regions in the brain, along with the ability to individually track white matter fibres using Diffusion Tensor Imaging (DTI), it becomes apparent that

*Address correspondence to this author at the CADE Clinic, Department of Psychiatry, Royal North Shore Hospital, St Leonards, Australia;

E-mail: jlagopoulos@med.usyd.edu.au neuroimaging today is able to provide unique insights into mentation and psychopathology and as a consequence aetiopathological models traditionally observed in psychiatric practice have shifted in focus from the mind to the brain. These advances have made it possible to investigate neural connections and the complex functional relationships between specific regions of the brain.

In this review we summarise progress that is being made in the field of imaging as it relates to psychiatric disorders and discuss the direction that future research is taking in tandem with its implications for clinical practice. As the discipline of psychiatry is broad and the imaging research being conducted extensive, the scope of this article precludes a comprehensive review across the breadth of the topic. Instead a detailed review of noteworthy findings, spanning a number of imaging modalities, across key psychiatric disorders is tabled.

\section{Mood Disorders}

Imaging research of mood disorders that encompasses major depression and bipolar disorder has been confounded by a lack of consistency in phenotypic grouping. Research to date has robustly identified limbic circuits in the generation and regulation of emotion and in particular frontostriatal circuits can be readily visualised using functional imaging techniques using paradigms that reliably modulate emotional tone.

Much of the early imaging research in mood disorders investigated structural differences in the brains of these patients and in particular attempted to differentiate gray from white matter. The majority of imaging studies that compared total brain volume of unipolar or bipolar disorder patients against that of healthy controls have found no significant differences [2-4]. The most consistent structural imaging 
finding in studies of patients with mood disorders is that of increased white matter hyperintensities (WMH) in unipolar patients $[5,6]$. WMH are changes identified on the MRI scan that signify a change of water content in the brain. A similar finding is noted in patients with bipolar disorder especially in subcortical gray, periventricular and deep white matter [6, 7]. However, signal hyperintensities lack anatomical and diagnostic specificity. They occur with ageing and are found in a variety of neuropsychiatric disorders including schizophrenia and dementia, hence their pathophysiological significance remains unclear. Volumetric brain studies suggest that there is a reduction in prefrontal cortical volume in unipolar depressed patients when compared to healthy controls [2, 8-11]. Further, structural MRI voxel-based morphometric analysis in patients with chronic treatmentresisitant depression [12], has identified reduced temporal lobe gray matter density in the anterior hippocampus. In bipolar disorder, temporal lobe changes are far less consistent with reports of both decreases [13] and increases $[10,14]$. Amygdala enlargement however, is a more consistent finding $[15,16]$ which supports its putative role in emotional processing. Thus, temporal lobe changes seem to distinguish unipolar and bipiolar disorder such that the hippocampus is reduced in size in unipolar depressed patients but largely unaltered in patients with bipolar disorder, whereas the reverse is true as regards the amygdala. In addition to cortical changes, striatal pathology is strongly implicated in the pathophysiology of mood disorders. Recent studies suggest reductions in size and changes in morphology of the anterior cingulate architecture in bipolar disorder [17].

Apart from structural brain changes, patients with mood disorders also exhibit functional abnormalities. A consistent finding in Cerebral Blood Flow (CBF) studies of mood disorder patients is that of decreased activity in anterior paralimbic as well as in additional cortical regions in response to paradigms that employ emotional stimuli. In major depression this finding of diminished blood flow reverses with successful treatment of the illness. Further, in depressed bipolar patients there is additional increased subcortical paralimbic metabolism [18] and together these findings support a limbic-cortical dysregulation model of depression [19]. This model posits dorsal neocortical hypofunction that precipitates ventral paralimbic overactivity along with reciprocity in this inverse relationship.

Proton spectroscopy studies in depressed patients have found modest increases in the concentration of choline within the basal ganglia and anterior cingulate [20]. These changes are more pronounced in patients that respond to antidepressants and although in need of further replication, it is likely that basal ganglia choline concentration is moodstate dependant [21]. Proton spectroscopy studies of bipolar patients also suggest state related changes in metabolism. Specifically, studies report diminished dorsolateral prefrontal $\mathrm{N}$-Acetyl-Aspartate (NAA) and creatine that indicates a decrease in neuronal density $[22,23]$. Basal ganglia NAA is also diminished in patients with bipolar disorder, especially in patients with a late onset of illness and more fine-tuned MRS studies have identified a reduction of orbito-frontal gray matter NAA [24]. It is well established that the neurotransmitter serotonin is strongly implicated in the pathophysiology of mood disorders and investigations have been significantly facilitated with the introduction of Positron Emission Tomography (PET) imaging and the development of specific ligands [25]. More specifically, the serotonin transporter (SERT) is strongly linked to the pathophysiology of depression [26] and its in vivo availability can be determined using selective PET radioligands that image the human 5HT transporter site. Binding to the serotonin transporter, serves as a proxy for the level of intrasynaptic serotonin, such that diminished binding is indicative of a decrease in intrasynaptic serotonin, essentially denoting diminished serotonergic functioning [27]. A recent study that utilising a selective PET radioligand for the human 5HT transporter site compared unipolar and bipolar patients that were antidepressant naïve with healthy controls. This study found significantly increased SERT availability in the thalamus of patients as compared to controls [28] suggesting that the thalamus is involved in the pathophysiology of mood disorders. A separate study that used the same PET ligand to examine drug-free depressed patients found significantly greater right cingulate and left frontal 5HT transporter site binding as compared with healthy controls [29]. PET studies in patients with bipolar and major depression examining $5-\mathrm{HT}_{1 \mathrm{~A}}$ receptor function have found significant reductions in receptor binding in limbic, frontal and temporal cortical regions [30]. Similarly, $5-\mathrm{HT}_{2 \mathrm{~A}}$ receptor PET studies also report a decrement in patients with major depression relative to controls $[31,32]$ however, interestingly some studies find no difference [33].

Functional MRI has revolutionised the manner in which brain function is studied by providing an avenue for examining the workings of the brain. Functional MRI methodology is particularly useful in psychiatry for investigating aberrant circuitry involved in processing emotion and in recent years it has been increasingly used to investigate the mood-disordered brain. With respect to unipolar depression, fMRI studies which have employed stimuli with positive and negative valences have consistently reported decreased BOLD activations in the dorsal prefrontal cortex (BA9) that is reversed with effective treatment [34, 35]. Responses in this region have also been identified in healthy subjects during memory driven sadness and happiness suggesting that the right prefrontal cortex (BA9) is an important destination for limbic projections and that it perhaps plays a modulating role in mood states [36]. Specifically, responses in BA9 indicate emotional awareness that focuses attention to internal emotional states [37] and involves greater cognitive emotional processing. The anterior cingulate is another region that appears to be integral to emotional decision making and is functionally pivotal to the experience of emotion, perhaps by virtue of its reciprocal interconnections to the orbital frontal cortex, amygdala and insula [38]. Functional activation and resting-state treatment studies [35, 36] along with neuropsychological and lesion studies [39] strongly implicate the sub-genual cingulate (BA25) in the pathogenesis of clinical depression, a region that is activated by autobiographical script-induced sadness $[35,40]$. However, the latter is not definitive as it may simply process the cognitive aspects of internally generated emotion rather than sadness per se.

In addition to the anterior cingulate, the amygdala is also of great interest as it has been shown to be activated across a range of functional imaging studies including those related to 
the detection of environmental threat and the experience of fear [41]. It is possible that its function is more general, determining for instance the dangerousness of affective stimuli or indeed their salience in respective of valence. Hence, in addition to fearful and averse stimuli [42] sad and happy affect [43] can also produce amygdala activation in functional imaging experiments in healthy volunteers and patients with major depression and bipolar disorder [44].

In examining bipolar disorder, fMRI studies have shown reduced activation notably in the left rostral ventral prefrontal cortex [45], and interestingly, hippocampal [42] and amygdala activation [43] have been found in both unipolar and bipolar patients resulting in a pattern of diminished prefrontal activation in major depression, and state-related subcortical activation in bipolar disorder [43, $46,47]$.

It is now well established that mood disorders are characterised by functional abnormalities that are readily identifiable using fMRI. However, the significance and interpretation of these observed changes within a clinical context is complicated partly because of the effects of medication, but also because of the complexity of emotional networks and our incomplete understanding of the integration of these networks with higher cognitive processes.

\section{Schizophrenia}

Converging neuropathological evidence from macroscopic and histological brain studies suggest that schizophrenia is a neurodevelopmental disorder. Functionally, there are distinct neurocognitive deficits, the neuropsychological underpinnings of which have been well described in the literature. Schizophrenia has therefore been an ideal candidate for both structural and functional imaging research. In addition, the dominant dopamine hypothesis that has driven drug development in schizophrenia has fostered imaging receptor studies that have examined many aspects of dopaminergic function.

Structural MRI has been particularly useful in schizophrenia research. In these patients WMHs are common and occur especially in late onset schizophrenia [48]. On the other hand, reductions in prefrontal cortical white matter volume have also been found in first-episode schizophrenia as well as twin studies and these changes have been associated with negative symptoms [49]. Early volumetric studies of schizophrenia patients reported enlarged lateral cerebral ventricles and initially, these changes were thought to be associated with diffuse brain atrophy. However, more recently it has been established that these changes associated with focal decreases in brain parenchyma in specific brain regions and nuclei, such as the putamen, thalamus and superior temporal gyrus [50]. This finding is further supported by a study that identified diminished mediodorsal and pulvinar thalamic nuclei volume in schizophrenia [51]. More recently, Diffusion Tensor Imaging (DTI) has further delineated structural deficits in schizophrenia. One group for instance has localised executive functional deficits to the left cingulate bundle, and declarative-episodic verbal memory deficits to the left uncinate fasciculus, in patients with schizophrenia [52]. In this regard DTI has been usefully applied to corroborate structural imaging findings with white matter tract changes occurring in prefrontal and temporal brain regions [53].

In the past, PET and SPECT studies have used amphetamine-induced reduction of raclopride binding to identify changes in dopamine D2 receptors. These neurotransmitter function studies show that there is increased dopamine release in schizophrenia corroborating the dopamine hypothesis theory [54]. Further, spectroscopy studies in schizophrenia have consistently reported a reduction in frontal and temporal cortex NAA concentrations suggesting neuronal loss that is in keeping with a localised reduction of gray matter [55].

A recent ${ }^{18}$ F-FDG PET study of glucose metabolism examined thalamic sub-divisions in patients with schizophrenia in 41 unmedicated patients and compared these with 60 matched healthy controls. The results of the study found significantly diminished glucose metabolism in the mediodorsal nucleus and centromedian nucleus of the thalamus in patients with schizophrenia compared to controls, but interestingly in the pulvinar it was significantly enhanced. A further finding from this study was that diminished mediodorsal nucleus glucose metabolism correlated with negative symptoms whereas diminished pulvinar glucose metabolism correlated with positive symptoms and hallucinations [56]. The partitioning of thalamic regions with contrasting changes in glucose metabolism and the correlation of these abnormalities to clinical phenomenology in this study demonstrates how sophisticated neuroimaging can inform our understanding of complex psychiatric illnesses such as schizophrenia.

In this context fMRI has also been useful in examining frontal lobe dysfunction that is thought to be at the core of schizophrenia. A consistent finding across a number of imaging modalities in schizophrenia has been that of hypofrontality, namely an inability to generate a frontal cerebral response to a specific task [57, 58]. This hypofrontality on fMRI is consistent with early PET and SPECT findings and some evidence suggests that it can to a degree be reversed, as shown by a verbal working memory fMRI study [57]. However, in recent times the notion that hypofrontality may constitute a trait marker has been challenged with several studies now reporting increased prefrontal activity in patients with schizophrenia [59, 60] particularly in studies which have employed the use of parametric-type experimental paradigms where experimental load is modulated $[60,61]$. The reasons for the disparity in results observed in the prefrontal cortex may in part be due to experimental factors and it seems that the observed increased in activity may relate to the recruitment for additional resources in response to increasing load, a process that may not be occurring equally in healthy subjects as existing resources may be adequately coping with the increased demand. Other factors which can't be excluded entirely but are difficult to verify may include the heterogeneity of the patients sample but also the number of included subjects. In other studies patients with schizophrenia have been shown in fMRI studies to have reduced activation in the left temporal lobe, right frontal lobe and left cerebellum.

More recently momentum has shifted to the field of genetics-imaging. This relatively new domain examines a 
number of key genes and their likely contributions to the cognitive symptoms experienced by schizophrenia patients and examining the interplay between genetics and behaviour has provided further insights into the pathophysiology of schizophrenia. The COMT gene has received significant attention in part due to its functional validity thus making it an obvious target for neuroimaging studies [62]. Several fMRI studies have reported a robust relationship between COMT polymorphisms and prefrontal activity including working memory performance to the point where the COMT genotype can predict performance on prefrontal function $[63$, 64]. Whilst the exact mechanism by which the COMT genotype modulates prefrontal activity has yet to be elucidated, the hypothesis is that the COMT Met allele together with higher synaptic dopamine concentrations results in a dysregulation of local GABA and glutamate transmission to the prefrontal cortex. The glutamatergic neurotransmitter system has also received recent attention with the particular focus on postsynaptic markers [65]. It has been suggested that the polymorphisms in the GRM3 gene (which encodes the metabotropic glutamate receptor), may be associated with cognitive changes observed in schizophrenia, and this may be mediated via altered glutamate transmission. However, as no single polymorphism has been identified in schizophrenia, this may imply that the GRM3 gene exerts its effects via the regulation of other glutamate-related proteins rather than via a direct route [66]. The G72 gene, associated with glutamate function, has also been the subject of several investigations and whilst numerous significant genotype-by-diagnosis interactions for cognitive measures have been reported [67], a lack of association between the effects of G72 SNPs on the risk of schizophrenia and cognitive impairment limits its interpretation with regards to the manifestation of cognitive impairments in this group.

\section{Post Traumatic Stress Disorder (PTSD)}

PTSD is caused by exposure to extreme trauma in which the person has experienced an event or a threat to the physical integrity of that person or others. The characteristic response involves intense fear, helplessness or horror. The pathophysiology of PTSD is linked to four mechanisms that include the fear response, a failure of fear extinction, behavioural sensitisation and memory problems. The structures involved in these processes include the sensory cortex, the dorsal thalamus, the lateral and central nucleus of the amygdala, the medial prefrontal cortex and hippocampus. These brain structures can be readily accessed using a variety of imaging techniques and the phenomenology of PTSD clearly lends itself to neurocognitive scrutiny.

Several studies have reported structural changes in PTSD patients involving the anterior cingulate cortex and the hippocampus [68]. The latter is reduced in size by a number of types of trauma including physical and sexual abuse [69]. However, despite these changes a causal relationship between reductions in hippocampal volume and a traumatic stressor is difficult to substantiate. Structural MRI studies of patients with PTSD have focussed on the hippocampus with few specifically examining the amygdala.

In contrast functional studies including PET, SPECT and fMRI have been particularly useful in investigating and understanding the pathophysiology of PTSD. Converging evidence from these studies suggests a pattern of altered metabolic activity in the medial prefrontal cortex-amygdala circuit [70]. The most consistent findings have been those of increased amygdala and decreased medial prefrontal cortex activation [71] whereas functional imaging responses in the hippocampus and parahippocampal gyrus have been much less consistent [69]. However, overall the findings support the hypothesis that there are two acute trauma response subtypes, one that is mainly dissociative and the other that is characterised by hyperarousal and intrusions [72].

\section{DISCUSSION}

Over the past decade, imaging has made a discernable impact upon our understanding of the neural processes that form the basis of psychiatric disorders. Currently the majority of psychiatric imaging is research-orientated. However, it is important to note its enormous clinical potential in psychiatry. Equally it is important to acknowledge the rapid advances that have been made in the research realm in a comparatively short period of time and the impact this has had on our understanding of the neural processes that form the basis of psychiatric disorders. Such is our understanding that in a relatively short period of time this knowledge has translated into a clinical domain and currently is used for in neurosurgical planning for severe, treatment resistant neuropsychiatric disease [73, 74] where information gleaned from imaging studies examining structure-function relationships provides anatomical target sites for restorative neurosurgical interventions. Given this rate of progress it seems inevitable that imaging will at some point in the near future be incorporated into routine clinical investigations alongside psychiatric mental state examination and neuropsychological evaluation. It is important however, to check our enthusiasm as at present imaging is somewhat limited in what it can offer clinically. Many structural and functional findings, for instance, relate to comparisons across groups of subjects and have little relevance to an individual with a psychiatric disorder. In reality imaging is just beginning to categorise the phenomena of the brain. This is perhaps not surprising since the neurobiology of cognition and the neural substrates of behaviour are by design complex and do not lend themselves easily to scientific examination. Added to this are the complexities of clinical populations such as the effects of medication and co-morbidity across psychiatric disorders.

A critical question in neuroimaging research of mood disorders is that of whether imaging findings are state or trait related. Trait related changes have the potential to provide a biological marker of the illness, which in practice may precede the manifestation of symptoms, and state-related changes offer potential means for monitoring treatment response. For schizophrenia, the challenges lie in the ability to combine complimentary imaging with neurochemical and genetic probes. It is clear that multiple genes that individually have small effect but through interactions exert significant influence mediate the risk of disease for many psychiatric disorders. Research that combines imaging and genetics may provide a powerful means of 'genotyping' patients at risk. Finally, in PTSD there is increased optimism that imaging may shed light on the aberrant brain circuits that subserve the illness and how changes in the functioning of these brain regions modulates the suppression of 
memories. Insights into the workings of this neural circuitry may help in the development of new treatment strategies.

\section{REFERENCES}

1] Malhi GS, Valenzuela M, Wen W, Sachdev P. Magnetic resonance spectroscopy and its applications in psychiatry. Aust N Z J Psychiatry 2002; 36(1): 31-43.

[2] Coffey CE, Wilkinson WE, Weiner RD, et al. Quantitative cerebral anatomy in depression. A controlled magnetic resonance imaging study. Arch Gen Psychiatry 1993; 50(1): 7-16.

[3] Dupont RM, Butters N, Schafer K, Wilson T, Hesselink J, Gillin JC. Diagnostic specificity of focal white matter abnormalities in bipolar and unipolar mood disorder. Biol Psychiatry 1995; 38(7): 482-6.

[4] Hoge EA, Friedman L, Schulz SC. Meta-analysis of brain size in bipolar disorder. Schizophr Res 1999; 37(2): 177-81.

[5] Altshuler LL, Curran JG, Hauser P, Mintz J, Denicoff K, Post R. T2 hyperintensities in bipolar disorder: magnetic resonance imaging comparison and literature meta-analysis. Am J Psychiatry 1995; 152(8): 1139-44.

[6] Drevets WC, Price JL, Simpson JR, et al. Subgenual prefrontal cortex abnormalities in mood disorders. Nature 1997; 386(6627): 824-7.

[7] Moore PB, El-Badri SM, Cousins D, et al. White matter lesions and season of birth of patients with bipolar affective disorder. Am J Psychiatry 2001; 158(9): 1521-4.

[8] Bremner JD, Narayan M, Anderson ER, Staib LH, Miller HL, Charney DS. Hippocampal volume reduction in major depression. Am J Psychiatry 2000; 157(1): 115-8.

[9] Krishnan KR, McDonald WM, Escalona PR, et al. Magnetic resonance imaging of the caudate nuclei in depression. Preliminary observations. Arch Gen Psychiatry 1992; 49(7): 553-7.

[10] Kumar A, Jin Z, Bilker W, Udupa J, Gottlieb G. Late-onset minor and major depression: early evidence for common neuroanatomical substrates detected by using MRI. Proc Natl Acad Sci USA 1998; 95(13): 7654-8

[11] Shah PJ, Ebmeier KP, Glabus MF, Goodwin GM. Cortical grey matter reductions associated with treatment-resistant chronic unipolar depression. Controlled magnetic resonance imaging study. Br J Psychiatry 1998; 172: 527-32.

[12] Schlaepfer TE, Harris GJ, Tien AY, et al. Decreased regional cortical gray matter volume in schizophrenia. Am J Psychiatry 1994; 151(6): 842-8.

[13] Hauser P, Altshuler LL, Berrettini W, Dauphinais ID, Gelernter J, Post RM. Temporal lobe measurement in primary affective disorder by magnetic resonance imaging. J Neuropsychiatr Clin Neurosci 1989; 1(2): 128-34.

[14] Altshuler LL, Bartzokis G, Grieder T, et al. An MRI study of temporal lobe structures in men with bipolar disorder or schizophrenia. Biol Psychiatry 2000; 48(2): 147-62.

[15] Hirayasu Y, McCarley RW, Salisbury DF, et al. Planum temporale and Heschl gyrus volume reduction in schizophrenia: a magnetic resonance imaging study of first-episode patients. Arch Gen Psychiatry 2000; 57(7): 692-9.

[16] Lenze EJ, Sheline YI. Absence of striatal volume differences between depressed subjects with no comorbid medical illness and matched comparison subjects. Am J Psychiatry 1999; 156(12): 1989-91.

[17] Chen X, Wen W, Malhi GS, Ivanovski B, Sachdev PS. Regional gray matter changes in bipolar disorder: a voxel-based morphometric study. Aust N Z J Psychiatry 2007; 41(4): 327-36.

[18] Mayberg HS, Brannan SK, Mahurin RK, et al. Cingulate function in depression: a potential predictor of treatment response. Neuroreport 1997; 8(4): 1057-61.

[19] Mayberg HS. Limbic-cortical dysregulation: a proposed model of depression. J Neuropsychiatr Clin Neurosci 1997; 9(3): 471-81.

[20] Winsberg ME, Sachs N, Tate DL, Adalsteinsson E, Spielman D, Ketter TA. Decreased dorsolateral prefrontal N-acetyl aspartate in bipolar disorder. Biol Psychiatry 2000; 47(6): 475-81.

[21] Renshaw PF, Lafer B, Babb SM, et al. Basal ganglia choline levels in depression and response to fluoxetine treatment: an in vivo proton magnetic resonance spectroscopy study. Biol Psychiatry 1997; 41(8): 837-43.
[22] Cecil KM, DelBello MP, Morey R, Strakowski SM. Frontal lobe differences in bipolar disorder as determined by proton MR spectroscopy. Bipolar Disord 2002; 4(6): 357-65.

[23] Hamakawa H, Kato T, Murashita J, Kato N. Quantitative proton magnetic resonance spectroscopy of the basal ganglia in patients with affective disorders. Eur Arch Psychiatr Clin Neurosci 1998; 248(1): 53-8.

[24] Davanzo P, Thomas MA, Yue K, et al. Decreased anterior cingulate myo-inositol/creatine spectroscopy resonance with lithium treatment in children with bipolar disorder. Neuropsychopharmacology 2001; 24(4): 359-69.

[25] Hesse S, Barthel H, Schwarz J, Sabri O, Muller U. Advances in in vivo imaging of serotonergic neurons in neuropsychiatric disorders. Neurosci Biobehav Rev 2004; 28(6): 547-63.

[26] Parsey RV, Mann JJ. Applications of positron emission tomography in psychiatry. Semin Nucl Med 2003; 33(2): 129-35.

[27] Ichimiya T, Suhara T, Sudo Y, et al. Serotonin transporter binding in patients with mood disorders: a PET study with $\left[{ }^{11} \mathrm{C}\right](+)$ McN5652. Biol Psychiatry 2002; 51(9): 715-22.

[28] Drevets WC. Prefrontal cortical-amygdalar metabolism in major depression. Ann N Y Acad Sci 1999; 877: 614-37.

[29] Reivich M, Amsterdam J, Brunswick D, et al. PET brain imaging with $\left[{ }^{11} \mathrm{C}\right](+) \mathrm{McN} 5652$ shows increased serotonin transporter availability in major depression. J Affect Disord 2004; 82: 321-7.

[30] Attar-Levy D, Martinot JL, Blin J, et al. The cortical serotonin2 receptors studied with positron-emission tomography and [18F]setoperone during depressive illness and antidepressant treatment with clomipramine. Biol Psychiatry 1999; 45(2): 180-6.

[31] Meltzer CC, Price JC, Mathis CA, et al. PET imaging of serotonin type $2 \mathrm{~A}$ receptors in late-life neuropsychiatric disorders. Am J Psychiatry 1999; 156(12): 1871-8.

[32] Meyer JH, Kapur S, Houle S, et al. Prefrontal cortex 5-HT2 receptors in depression: an $[18 \mathrm{~F}]$ setoperone PET imaging study. Am J Psychiatry 1999; 156(7): 1029-34.

[33] Mayberg HS, Liotti M, Brannan SK, et al. Reciprocal limbiccortical function and negative mood: converging PET findings in depression and normal sadness. Am J Psychiatry 1999; 156(5): 675-82.

[34] George MS, Ketter TA, Parekh PI, Horwitz B, Herscovitch P, Post RM. Brain activity during transient sadness and happiness in healthy women. Am J Psychiatry 1995; 152(3): 341-51.

[35] Lane RD, Chua PM, Dolan RJ. Common effects of emotional valence, arousal and attention on neural activation during visual processing of pictures. Neuropsychologia 1999; 37(9): 989-97.

[36] Lane RD, Reiman EM, Bradley MM, et al. Neuroanatomical correlates of pleasant and unpleasant emotion. Neuropsychologia 1997; 35(11): 1437-44.

[37] Critchley H. Emotion and its disorders. Br Med Bull 2003; 65: 3547.

[38] Devinsky O, Morrell MJ, Vogt BA. Contributions of anterior cingulate cortex to behaviour. Brain 1995; 118(Pt 1): 279-306.

[39] Happe F, Malhi GS, Checkley S. Acquired mind-blindness following frontal lobe surgery? A single case study of impaired 'theory of mind' in a patient treated with stereotactic anterior capsulotomy. Neuropsychologia 2001; 39(1): 83-90.

[40] Liotti M, Mayberg HS, Brannan SK, McGinnis S, Jerabek P, Fox PT. Differential limbic--cortical correlates of sadness and anxiety in healthy subjects: implications for affective disorders. Biol Psychiatry 2000; 48(1): 30-42.

[41] Morris JS, Friston KJ, Buchel C, et al. A neuromodulatory role for the human amygdala in processing emotional facial expressions. Brain 1998; 121(Pt 1): 47-57.

[42] Lawrence NS, Williams AM, Surguladze S, et al. Subcortical and ventral prefrontal cortical neural responses to facial expressions distinguish patients with bipolar disorder and major depression. Biol Psychiatry 2004; 55(6): 578-87.

[43] Surguladze S, Brammer MJ, Keedwell P, et al. A differential pattern of neural response toward sad versus happy facial expressions in major depressive disorder. Biol Psychiatry 2005; 57(3): 201-9.

[44] Malhi GS, Lagopoulos J, Owen AM, Yatham LN. Bipolaroids: functional imaging in bipolar disorder. Acta Psychiatr Scand Suppl 2004; (422): 46-54.

[45] Blumberg HP, Leung HC, Skudlarski P, et al. A functional magnetic resonance imaging study of bipolar disorder: state- and 
trait-related dysfunction in ventral prefrontal cortices. Arch Gen Psychiatry 2003; 60: 601-9.

[46] Lagopoulos J, Malhi GS. A functional magnetic resonance study of emtional stoop in euthymic bipolar disorder. Neuroreport 2007; 18(15): 1583-7.

[47] Malhi GS, Lagopoulos J, Ward PB, et al. Cognitive generation of affect in bipolar depression: an fMRI study. Eur J Neurosci 2004; 19(3): 741-54.

[48] Rivkin P, Kraut M, Barta P, Anthony J, Arria AM, Pearlson G. White matter hyperintensity volume in late-onset and early-onset schizophrenia. Int J Geriatr Psychiatry 2000; 15(12): 1085-9.

[49] Davis KL, Stewart DG, Friedman JI, et al. White matter changes in schizophrenia: evidence for myelin-related dysfunction. Arch Gen Psychiatry 2003; 60(5): 443-56.

[50] Gaser C, Nenadic I, Buchsbaum BR, Hazlett EA, Buchsbaum MS. Ventricular enlargement in schizophrenia related to volume reduction of the thalamus, striatum, and superior temporal cortex. Am J Psychiatry 2004; 161(1): 154-6.

[51] Byne W, Buchsbaum MS, Kemether E, et al. Magnetic resonance imaging of the thalamic mediodorsal nucleus and pulvinar in schizophrenia and schizotypal personality disorder. Arch Gen Psychiatry 2001; 58(2): 133-40.

[52] Nestor PG, Kubicki M, Gurrera RJ, et al. Neuropsychological correlates of diffusion tensor imaging in schizophrenia. Neuropsychology 2004; 18(4): 629-37.

[53] Shin YW, Kwon JS, Ha TH, et al. Increased water diffusivity in the frontal and temporal cortices of schizophrenic patients. Neuroimage 2006; 30(4): 1285-91.

[54] Laruelle M, Abi-Dargham A. Dopamine as the wind of the psychotic fire: new evidence from brain imaging studies. J Psychopharmacol 1999; 13(4): 358-71.

[55] Sigmundsson T, Maier M, Toone BK, et al. Frontal lobe Nacetylaspartate correlates with psychopathology in schizophrenia: a proton magnetic resonance spectroscopy study. Schizophr Res 2003; 64(1): 63-71.

[56] Hazlett EA, Buchsbaum MS, Kemether E, et al. Abnormal glucose metabolism in the mediodorsal nucleus of the thalamus in schizophrenia. Am J Psychiatry 2004; 161(2): 305-14.

[57] Honey GD, Bullmore ET, Soni W, Varatheesan M, Williams SC, Sharma T. Differences in frontal cortical activation by a working memory task after substitution of risperidone for typical antipsychotic drugs in patients with schizophrenia. Proc Natl Acad Sci USA 1999; 96(23): 13432-7.

[58] Weiss EM, Siedentopf C, Golaszewski S, et al. Brain activation patterns during a selective attention test--a functional MRI study in healthy volunteers and unmedicated patients during an acute episode of schizophrenia. Psychiatry Res 2007; 154(1): 31-40.

[59] Karlsgodt KH, Sanz J, van Erp TG, Bearden CE, Nuechterlein KH, Cannon TD. Re-evaluating dorsolateral prefrontal cortex activation during working memory in schizophrenia. Schizophr Res 2009; 108(1-3): 143-50.

[60] Potkin SG, Turner JA, Brown GG, et al. Working memory and DLPFC inefficiency in schizophrenia: the FBIRN study. Schizophr Bull 2009; 35(1): 19-31.

[61] Woodward ND, Waldie B, Rogers B, Tibbo P, Seres P, Purdon SE. Abnormal prefrontal cortical activity and connectivity during response selection in first episode psychosis, chronic schizophrenia, and unaffected siblings of individuals with schizophrenia. Schizophr Res 2009; 109(1-3): 182-90.

[62] Roffman JL, Weiss AP, Goff DC, Rauch SL, Weinberger DR. Neuroimaging-genetic paradigms: a new approach to investigate the pathophysiology and treatment of cognitive deficits in schizophrenia. Harv Rev Psychiatry 2006; 14(2): 78-91.

[63] Egan MF, Goldberg TE, Kolachana BS, et al. Effect of COMT Val108/158 Met genotype on frontal lobe function and risk for schizophrenia. Proc Natl Acad Sci USA 2001; 98(12): 6917-22.

[64] Weinberger DR, Egan MF, Bertolino A, et al. Prefrontal neurons and the genetics of schizophrenia. Biol Psychiatry 2001; 50(11): $825-44$.

[65] Tsai G, Coyle JT. Glutamatergic mechanisms in schizophrenia Annu Rev Pharmacol Toxicol 2002; 42: 165-79.

[66] Egan MF, Straub RE, Goldberg TE, et al. Variation in GRM3 affects cognition, prefrontal glutamate, and risk for schizophrenia. Proc Natl Acad Sci USA 2004; 101(34): 12604-9.

[67] Goldberg TE, Straub RE, Callicott JH, et al. The G72/G30 gene complex and cognitive abnormalities in schizophrenia. Neuropsychopharmacology 2006; 31(9): 2022-32.

[68] Geuze E, Vermetten E, Bremner JD. MR-based in vivo hippocampal volumetrics: 2. Findings in neuropsychiatric disorders. Mol Psychiatry 2005; 10(2): 160-84.

[69] Bremner JD, Vythilingam M, Vermetten E, et al. MRI and PET study of deficits in hippocampal structure and function in women with childhood sexual abuse and posttraumatic stress disorder. Am J Psychiatry 2003; 160(5): 924-32.

[70] Francati V, Vermetten E, Bremner JD. Functional neuroimaging studies in posttraumatic stress disorder: review of current methods and findings. Depress Anxiety 2007; 24(3): 202-18.

[71] Britton JC, Phan KL, Taylor SF, Fig LM, Liberzon I. Corticolimbic blood flow in posttraumatic stress disorder during script-driven imagery. Biol Psychiatry 2005; 57(8): 832-40.

[72] Lanius RA, Bluhm R, Lanius U, Pain C. A review of neuroimaging studies in PTSD: heterogeneity of response to symptom provocation. J Psychiatr Res 2006; 40(8): 709-29.

[73] Aouizerate B, Cuny E, Bardinet E, et al. Distinct striatal targets in treating obsessive-compulsive disorder and major depression. $\mathrm{J}$ Neurosurg 2009; (in press).

[74] Malone DA Jr, Dougherty DD, et al. Deep brain stimulation of the ventral capsule/ventral striatum for treatment-resistant depression. Biol Psychiatry 2009; 65(4): 267-75. 